\title{
Development of Degassing Device for Transformer oil Based on Headspace Degassing Method
}

\author{
Zhibin Yin ${ }^{1 *}$, Chao Huo ${ }^{1}$, Haipeng Sun ${ }^{1}$ and Jing Chen ${ }^{1}$ \\ ${ }^{1}$ Beijing Smartchip Microelectronics Technology Co., Ltd, Dept. of Terminal Communication, Beijing, China
}

\begin{abstract}
By analyzing the type and content of gas in oil, the latent faults in equipment can be found as soon as possible, and the faults can be monitored at any time. In this paper, a degassing device based on headspace degassing method is designed. The oil circuit adopts the way of oil pump and battery valve to complete the oil inlet and oil discharge. Through stirring, the dissolved gas in the oil can reach the dynamic balance in the gas-liquid two-phase, so as to realize the rapid and effective separation of the gas in the oil. At the same time, after degassing, the oil can be sent back to the body. In addition, the degassing verification of the device is carried out, and the effect is good. In the next step, the device can be integrated with the gas detection equipment to form a sealed cabinet detection equipment, which is of great significance for the technical realization and engineering application of online monitoring and live detection of power equipment.
\end{abstract}

\section{Introduction}

Power transformer is one of the core equipment in the power system, which is very important in the production, transmission and consumption of power. The research on fault diagnosis method of power transformer is of decisive significance to improve the safety and reliability of power system operation. Through effective and reliable monitoring method, the latent faults existing in the transformer can be found in time, and the condition based maintenance can be realized to ensure the safety of power system. The methods to evaluate transformer status include traditional methods such as dissolved gas analysis in oil, dielectric loss detection, winding ratio measurement and temperature monitoring, and nontraditional methods such as partial discharge and on-line power factor measurement. Restricted by many factors such as detection cost, data interpretation, result reliability and application compatibility, dissolved gas analysis in oil is most widely used.

The purpose of oil-gas separation is to quickly, automatically and efficiently separate the fault characteristic gas dissolved in the oil, and to ensure the long life as far as possible without pollution. At present, the commonly used oil-gas separation methods at home and abroad are membrane permeation, vacuum degassing and head space degassing ${ }^{[1-3]}$. Among them, the membrane permeation method makes the dissolved characteristic gas gradually penetrate into the detection chamber, which has the problems of long equilibrium time, environmental temperature, and the installation mode; the vacuum degassing method adopts the negative pressure mode, which has the problems of gas re dissolution due to the influence of environmental conditions, and the degassing rate changes The headspace degassing method is a dynamic balance method, through a certain amount of stirring, the dissolved gas in the oil can reach the distribution balance in the gas-liquid two-phase, so as to realize the rapid and effective separation of the gas in the oil. Headspace degassing method is a dynamic equilibrium method, so there is basically no gas dissolution, and the degassing efficiency and degassing consistency are good, which is one of the main ways of oil and gas separation ${ }^{[4]}$. Based on the headspace degassing method, a degassing device for transformer oil is developed in this paper. The oil circuit adopts the way of oil pump and battery valve to realize oil inlet and oil discharge, which can return the oil to the body after the test. The device can be easily applied to the fault analysis and condition monitoring of power transformer. It has the characteristics of simple structure, good degassing consistency, low cost and easy to carry. At the same time, it can be integrated with the gas detection equipment to complete the process of automatic gas sampling, detection and analysis, which ensures the accuracy of the analysis results and the safety in the sampling process, and provides an effective means for the research and application of on-line detection.

\section{Technical principles}

The principle of headspace degassing is to make the dissolved gas in the oil reach the distribution equilibrium in the gas-liquid two-phase through certain stirring, so as to realize the rapid and effective separation of the gas in the oil. By measuring the concentration of each component in the gas phase, and according to the

\footnotetext{
*Corresponding author: yzb2702@163.com
} 
Ostwald system derived from the principle of equilibrium, the concentration of each component of dissolved gas in oil can be calculated. Ostwald coefficient is defined as:

$$
k_{i}=\frac{c_{o i}}{c_{g i}}
$$

Where $C_{o i}$ and $C_{g i}$ are the concentrations of component $i$ dissolved in oil and gas phase under equilibrium conditions respectively; $k_{i}$ is the Ostwald coefficient of component $i$.

When degassing is completed, the higher the concentration of the gas to be detected, the better, which is conducive to the detection of subsequent instruments. Meanwhile, the shorter the degassing time, the better. When the volume of oil sample is constant, the total mass of gas component $i$ is conserved.

$$
U_{o i} V_{o}=C_{o i}^{t} V_{0}+C_{g i}^{t} V_{g}
$$

Where $V_{o}$ and $V_{g}$ represent the volume of oil and gas and gas chamber respectively; $C_{o i}^{t}$ and $C_{g i}^{t}$ represents the concentration of gas component $i$ and gas phase component $i$ in the oil at time $t ; U_{o i}$ is the crude oil concentration of a component gas in the oil sample. Assuming $t \rightarrow \infty$, the equilibrium concentration of gas component $i$ can be obtained:

$$
c_{g i}=\frac{U_{o i}}{k_{i}+\frac{V_{g}}{V_{0}}}
$$

For a specific degassing device, with the increase of degassing time, the concentration of a component gas in the gas chamber increases until it tends to a stable value, and the growth rate gradually decreases with the passage of degassing time. Generally, if the concentration of the gas chamber is $90 \%$ of the theoretical equilibrium concentration, it can be considered that the gas-liquid two-phase has reached equilibrium.

$$
\tau=\frac{2.5}{\frac{1}{V_{0}}+\frac{k_{i}}{V_{g}} \cdot D_{i}}
$$

It can be seen from equations (3) and (4) that the equilibrium concentration is only related to the original concentration of a component gas in the oil sample and the volume ratio of the gas chamber to the oil sample. Appropriately reducing this ratio can improve the gas concentration at equilibrium time and obtain higher degassing rate. The specific structure of degassing device determines the time needed for gas-liquid equilibrium. The smaller the volume of gas chamber and oil sample is, the shorter the time needed to reach equilibrium is. Therefore, on the premise of meeting the requirements of detection instrument, the size of degassing device should be reduced as much as possible, and the appropriate volume ratio of gas chamber and oil sample should be selected to obtain relatively high degassing efficiency ${ }^{[5]}$. The ideal degassing curve is shown in Figure 1.

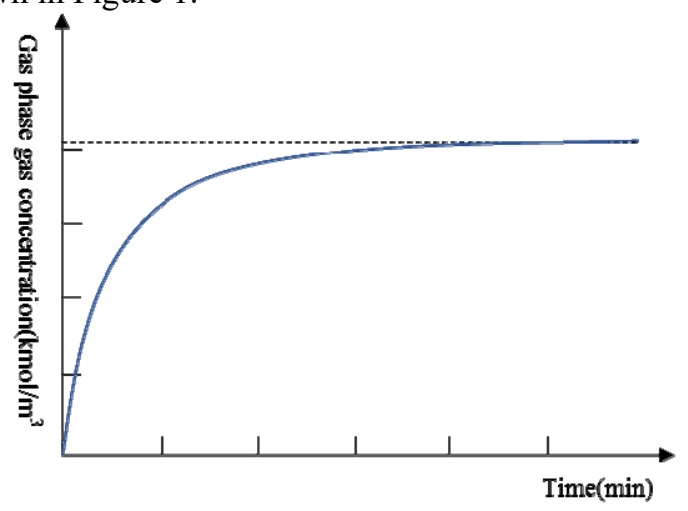

Fig. 1. Ideal degassing curve.

\section{System scheme}

It can be seen from the above technical principle that the degassing efficiency depends on the specific structure of the degassing device. According to the requirements of field test application, this paper designs an oil and gas degassing device with simple structure, easy to carry, and can be integrated with detection equipment. The system mainly includes pressure sensor, liquid level sensor, mixing motor, oil pump, gas pump, main control system, etc.

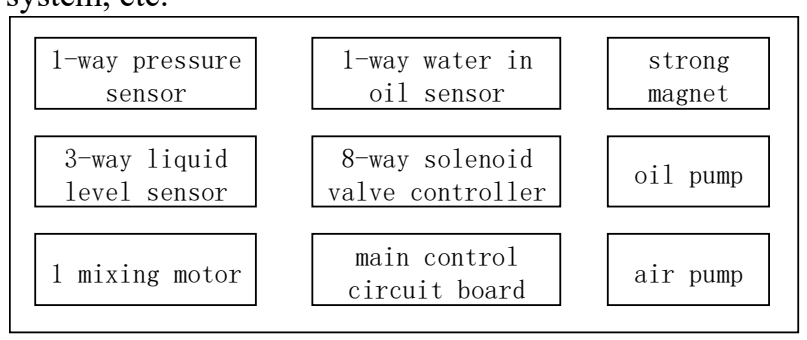

Fig.2. System composition of oil gas separation unit.

The device uses the headspace degassing method to make the dissolved gas in the oil reach the distribution equilibrium in the gas-liquid two phases through certain stirring, so as to realize the rapid and effective separation of the gas in the oil ${ }^{[6]}$.

The process of oil sample in degassing device mainly includes: transformer oil entering degassing device, circulating ventilation and continuous stirring, returning oil sample after degassing, separated gas entering gas storage chamber for storage, etc. Firstly, vacuum pump is used to pump the cylinder into vacuum state, then oil valve 3 and oil valve 4 are opened, other solenoid valves are closed, and insulating oil for test enters the cylinder through oil pump; then oil valve 1, 2, 3 and 4 are closed, and oil sample is stirred by magnetic stirrer to speed up oil-gas separation. The separated gas accumulates in the upper part of the cylinder. When the gas-liquid twophase reaches equilibrium, open the gas valve 2, and the gas enters the cylinder for sealing. Finally, after the completion of gas extraction, open oil valve 4 and close other valves to drain the insulating oil back to the 
transformer equipment. This not only ensures the reliable acquisition of the gas in the oil, but also can return the oil to the body after the test, ensuring the normal oil level of the equipment.

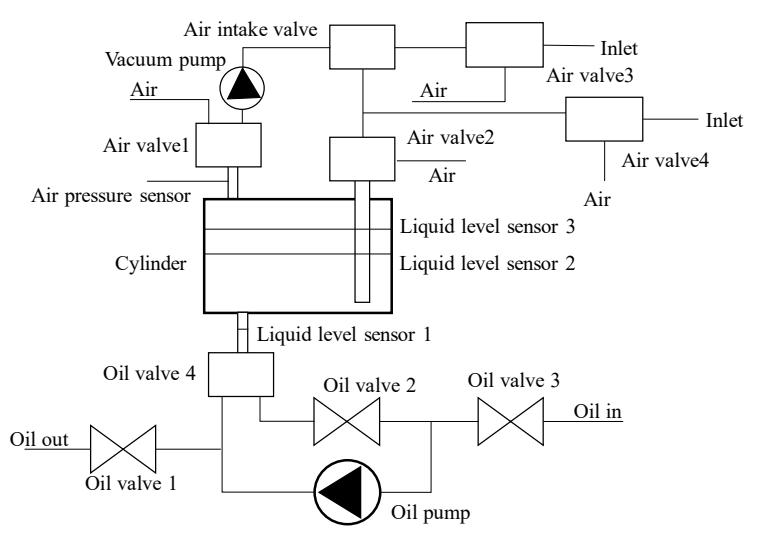

Fig.3. Working flow chart of degassing unit.

\section{4 system implementation}

The device mainly consists of bottom degassing part and main control system. The bottom degassing components include oil pump, air pump, solenoid valve, sensor and other components, which are controlled by the main control system. The main control system is the core part of the whole degassing device. Its main function is to send and dispatch various control parameters of the degassing device, and complete the real-time analysis and processing of the collected data. This distributed system architecture can simplify the system implementation complexity, reduce the design cost and risk, and improve the flexibility of design and use.

\subsection{Hardware design}

The hardware design scheme of the main control system is shown in Figure 4. The processor with high performance and low power consumption embedded arm Cortex-M0 core is adopted. The processor has rich resources and communication interfaces, which can meet the design requirements. The power supply part adopts DC $24 \mathrm{~V}$ power supply, which provides DC voltage for all levels of analogy and digital devices on board through power conversion; the analogy amplifier circuit realizes the acquisition and amplification of analogy quantity such as liquid level sensor and air pressure sensor, and uses 16 bit $\mathrm{ADC}$ digital to analogy conversion based on ltc1865 to complete the conversion of analogy quantity such as pressure and liquid level; the 8-way optocoupler circuit realizes gas filling and gas inlet The other two optocoupler circuits realize the startup and speed control of the mixing motor; the communication interface circuit includes RS485 circuit, RS232 circuit and JTAG circuit. RS485 circuit is used to communicate with upper computer, RS232 and JTAG are used to download and debug programs.

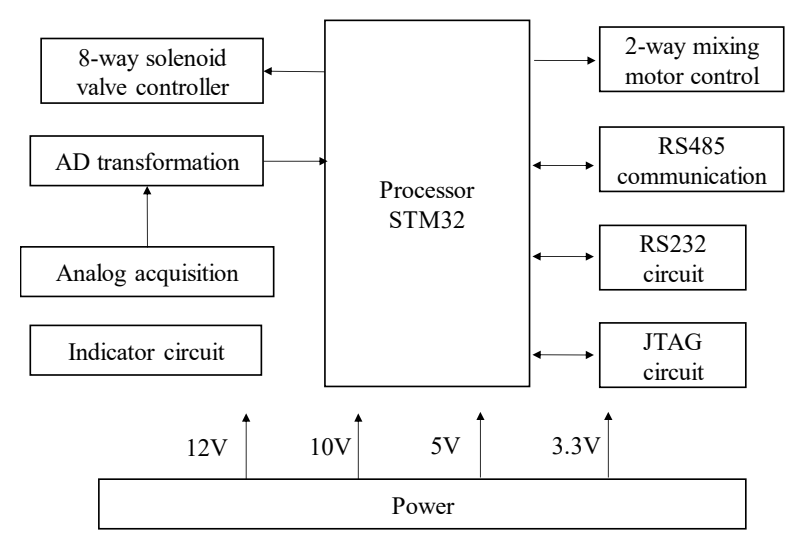

Fig.4. Hardware design of main control system.

\subsection{Software design}

The software part is divided into three threads, which run independently. The synchronization and communication between threads are realized through the internal interface, mainly including the main thread, acquisition thread and processing thread. As shown in Figure 5.

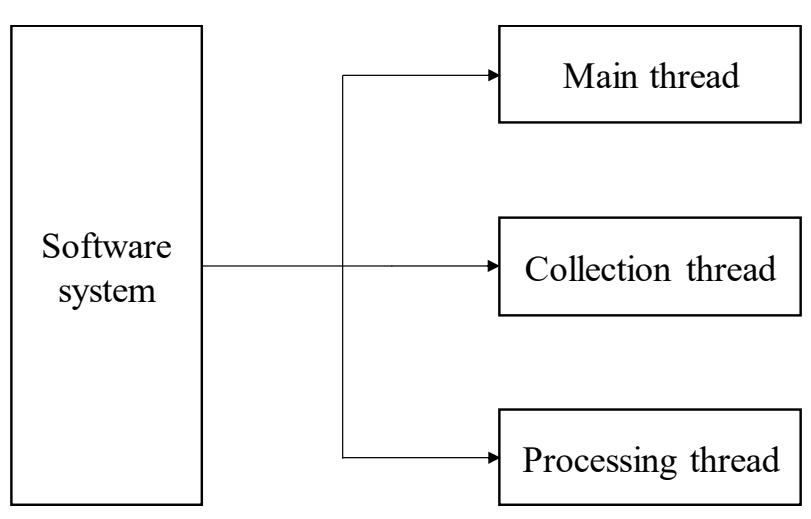

Fig.5. Software design of main control system.

1) The main functions of the main thread include: starting and closing degassing process; generating thread interrupt events; monitoring equipment operation, starting and closing acquisition and processing threads; browsing historical data; parameter setting and command issuing, etc.

2) The main functions of the acquisition thread include: driving the acquisition module to complete the analogy acquisition; saving the original data to the cache; generating the current acquisition completion event and starting the pending processing thread; generating the data band acquisition completion event and applying to the main thread for automatic shutdown.

3) The main functions of the processing thread include: reading the original data from the cache synchronously with the acquisition thread; feature suppression and feature extraction of the original data; data packet and data analysis, communication with the host computer, etc. 


\section{Field application}

After the successful development of the degassing device, the test verification was carried out in the laboratory of the substation maintenance center, and the degassing treatment and detection were carried out on the sample of the body oil of the II bus phase $\mathrm{C}$ reactor in the $500 \mathrm{kV}$ Daoguanhe substation. Firstly, the dissolved gas in the sample oil was degassed by the automatic degassing vibration instrument specified in the national standard GB / t17623-2017 ${ }^{[7]}$, and the degassed gas was analyzed by gas chromatograph Then the dissolved gas in the sample oil is degassed by the oil gas separation device described in this paper, and the degassed gas is also detected by gas chromatography; finally, the repeatability test is carried out, and the performance evaluation of the oil gas separation device is completed by comparing the test results ${ }^{[8-10]}$.

Table 1. Comparison table of test results.

\begin{tabular}{lcccccccccc}
\hline Degassing device & Time & $\begin{array}{c}\text { Degassing } \\
\text { time }\end{array}$ & $\mathrm{H}_{2}$ & $\mathrm{CO}$ & $\mathrm{CO}_{2}$ & $\mathrm{CH}_{4}$ & $\mathrm{C}_{2} \mathrm{H}_{4}$ & $\mathrm{C}_{2} \mathrm{H}_{6}$ & $\mathrm{C}_{2} \mathrm{H}_{2}$ & $\begin{array}{l}\text { Total } \\
\text { hydrocarbon }\end{array}$ \\
\hline $\begin{array}{l}\text { Automatic } \\
\text { degassing oscillator }\end{array}$ & $/$ & 30 & 10 & 1344 & 6099 & 50.3 & 10.4 & 12.2 & 0.2 & 73.1 \\
$\begin{array}{l}\text { Oil gas separation } \\
\text { unit }\end{array}$ & 1 & 25 & 8 & 1163 & 4984 & 48.7 & 10.1 & 11.4 & 0.2 & 70.4 \\
$\begin{array}{l}\text { Oil gas separation } \\
\text { unit }\end{array}$ & 2 & 25 & 9 & 1195 & 5027 & 48.8 & 10.1 & 11.5 & 0.2 & 70.6 \\
$\begin{array}{l}\text { Oil gas separation } \\
\text { unit }\end{array}$ & 3 & 25 & 11 & 1248 & 5439 & 49.3 & 10.0 & 11.6 & 0.2 & 71.2 \\
$\begin{array}{l}\text { Oil gas separation } \\
\text { unit }\end{array}$ & 4 & 25 & 9 & 1406 & 5130 & 49.1 & 9.9 & 11.6 & 0.2 & 70.7 \\
$\begin{array}{l}\text { Oil gas separation } \\
\text { unit }\end{array}$ & 5 & 25 & 7 & 1086 & 5289 & 46.2 & 8.2 & 11.3 & 0.2 & 65.9 \\
$\begin{array}{l}\text { Oil gas separation } \\
\text { unit }\end{array}$ & 6 & 25 & 8 & 1197 & 5385 & 47.6 & 9.9 & 12.1 & 0.3 & 69.8 \\
$\begin{array}{l}\text { Total hydrocarbon } \\
\text { RSD }\end{array}$ & & & & & $1.8 \%$ & & & & \\
\hline
\end{tabular}

The experimental results show that the degassing time and degassing efficiency of the device can meet the requirements of practical application. The degassing repeatability meets the requirement of RSD $\leqslant 5 \%$ of total hydrocarbon.

\section{Conclusion}

In this paper, the degassing device based on headspace degassing method is designed, which makes the dissolved gas in oil reach the distribution balance in the gas-liquid two-phase through certain stirring, so as to realize the rapid and effective separation of gas in oil. The field test shows that the degassing time, degassing efficiency and degassing consistency of the device can meet the requirements of practical application. The device has the characteristics of simple structure, portability and easy integration, which is of great significance to the technical realization and engineering application of online monitoring and live detection of power equipment, and has a good promotion prospect.

\section{Acknowledgment}

This work is supported by "National Key R\&D Program of China No.2018YFF01011800”.

\section{References}

1. Shuangzan R, Hao W, Xiaoli L, et al. ET, 19(2020)

2. Sheng X. Shandong IT, 9 (2018)
3. Dongliang L, Xinyan W. Jiangsu EE, 28(2009)

4. Chunyan S, Mingyue C. EM. 5(2014)

5. Renbiao Z, Wei W, Xibin H, et al. AOIC, 19 (2020)

6. Haowei H. Zhejiang EP, 35(2016)

7. GB/T17623-2017[S].

8. Xiuhua L. ET, 11(2016)

9. Qitian B. SCUT, (2013)

10. Xiao L. HPS, 4 (2017) 